\title{
Quartz Crystal Microbalances for Evaluating Gas Motion Differences between Dichlorosilane and Trichlorosilane in Ambient Hydrogen in a Slim Vertical Cold Wall Chemical Vapor Deposition Reactor
}

\author{
Mana Otani ${ }^{1}$, Toshinori Takahashi ${ }^{1}$, Hitoshi Habuka ${ }^{*}(\mathbb{D}$, \\ Yuuki Ishida ${ }^{2,3}$, Shin-Ichi Ikeda ${ }^{2,3}$, Shiro Hara2,3 \\ ${ }^{1}$ Department of Chemistry Applications and Life Science, Yokohama National University, Hodogaya, Yokohama, Japan \\ ${ }^{2}$ National Institutes of Advanced Science and Technology, Umezono, Tsukuba, Japan \\ ${ }^{3}$ Minimal Fab Development Association, Umezono, Tsukuba, Japan \\ Email: ^habuka-hitoshi-ng@ynu.ac.jp
}

How to cite this paper: Otani, M., Takahashi, T., Habuka, H., Ishida, Y., Ikeda, S.-I. and Hara, S. (2020) Quartz Crystal Microbalances for Evaluating Gas Motion Differences between Dichlorosilane and Trichlorosilane in Ambient Hydrogen in a Slim Vertical Cold Wall Chemical Vapor Deposition Reactor. Advances in Chemical Engineering and Science, 10, 190-200. https://doi.org/10.4236/aces.2020.103014

Received: May 28, 2020

Accepted: June 27, 2020

Published: June 30, 2020

Copyright $\odot 2020$ by author(s) and Scientific Research Publishing Inc. This work is licensed under the Creative Commons Attribution International License (CC BY 4.0).

http://creativecommons.org/licenses/by/4.0/

\begin{abstract}
A dichlorosilane gas and a trichlorosilane gas in ambient hydrogen were evaluated to show their different gas flow motions in a slim vertical cold wall chemical vapor deposition reactor for the Minimal Fab system. This evaluation was performed for improving and controlling the film qualities and the productivities, using two quartz crystal microbalances (QCM) installed at the inlet and exhaust of the chamber by taking into account that the QCM frequency corresponds to the real time changes in the gas properties. Typically, the time period approaching from the inlet to the exhaust was shorter for the trichlorosilane gas than that for the dichlorosilane gas. The trichlorosilane gas was shown to move like plug flow, while the dichlorosilane gas seemed to be well mixed in the entire chamber.
\end{abstract}

\section{Keywords}

Minimal Fab, Chemical Vapor Deposition Reactor, Quartz Crystal Microbalance, Silicon Epitaxial Growth, Trichlorosilane, Dichlorosilane

\section{Introduction}

Chemical Vapor Deposition (CVD) [1]-[6] is a useful technique for fabricating thin and functional material films for advanced electronics. For the CVD, vari- 
ous precursors and carrier gases having various gas properties are used. Because the gas density and viscosity may influence the gas motions in the chamber, any gas flow information influenced by the gas properties should be studied. Such an evaluation may contribute to improving and controlling the film qualities and the productivity.

For this purpose, the in situ and real time gas sensing technique [4] [5] [7] is significantly useful, such as that using the quartz crystal microbalance [7]-[12]. The QCM is sensitive to the slight changes in the properties of the gas mixture corresponding to the gas composition. Additionally, the QCM has been used in an ambient reactive gas, such as that containing hydrogen chloride gas, which was a byproduct of the silicon CVD using chlorosilanes [2] [6].

For this purpose, in our previous study [7], a quartz crystal microbalance (QCM) [8] [9] [10] [11] [12] was connected to the slim vertical cold wall CVD reactor designed for the Minimal Fab [12]-[18]. Because the Minimal Fab should achieve a high productivity using small wafers (12.5 $\mathrm{mm}$ diameter), the in situ measurement is expected for making the Minimal CVD process fast.

The QCM and the theoretical calculations showed the trend that the gas density influenced the gas motions in a chlorosilane-hydrogen system [7] [13]. Next, for obtaining further details, the gas motions at the inlet and exhaust should be real time measured and compared in detail.

In this study, the slim vertical cold wall CVD reactor equipped with two QCM sensors was used in order to quantitatively study the difference in the gas motion between two industrially-used chlorosilanes, such as the dichlorosilane $\left(\mathrm{SiH}_{2} \mathrm{Cl}_{2}\right.$, DCS) and trichlorosilane $\left(\mathrm{SiHCl}_{3}, \mathrm{TCS}\right)$ gases.

\section{Experimental Procedure}

Figure 1 shows the simplified configuration of CVD reactor used in this study. At the center position in the slim vertical quartz tube, a $12.5 \mathrm{~mm}$ (half inch)-diameter silicon wafer was placed on the rotating quartz rod. The infrared light came from three halogen lamps through the transparent quartz tube in order to heat the wafer. The halogen lamp voltages were $66-72 \mathrm{~V}$. The wafer temperature was near or below $900^{\circ} \mathrm{C}$ at which the typical silicon deposition rate was about $0.5 \mu \mathrm{m} \cdot \mathrm{min}^{-1}$.

The wafer rotation rate was $0-60 \mathrm{rpm}$. The trichlorosilane and dichlorosilane gases were introduced from the top of chamber in ambient hydrogen at atmospheric pressure. Using these gases, the silicon epitaxial film was formed on the silicon wafer surface by the overall chemical reactions [2] [6] as follows:

$$
\begin{aligned}
& \text { Trichlorosilane : } \mathrm{SiHCl}_{3}+\mathrm{H}_{2} \rightarrow \mathrm{Si}+3 \mathrm{HCl} \\
& \text { Dichlorosilane : } \mathrm{SiH}_{2} \mathrm{Cl}_{2}+\mathrm{H}_{2} \rightarrow \mathrm{Si}+2 \mathrm{HCl}
\end{aligned}
$$

Two QCM sensors (25 MHz) contained in a small box, as shown in Figure 1(b), were placed at the inlet and exhaust, as shown in Figure 1(a). The QCM box at the inlet measured the gases at room temperature before undergoing any 
chemical reactions. The gases after the silicon film formation were cooled to room temperature and measured by the QCM at the exhaust. The QCM sensors were initially experimentally confirmed not to have any influence due to the thermal condition in the chamber. The QCM frequency in this study was influenced by only two parameters, such as the gas properties in the gas phase [9] and the byproduct deposition on the surface [19]. Throughout the silicon epitaxial film deposition process, the QCM frequency was measured and recorded by a personal computer.

The process shown in Figure 2 was used for the silicon epitaxial film deposition. First, the silicon wafer surface was heated to temperatures higher than $1000^{\circ} \mathrm{C}$ in ambient hydrogen for removing the native oxide film and organic contaminations on the silicon wafer surface. Next, the silicon wafer temperature was adjusted to that for the silicon film deposition. The flow rates of the trichlorosilane and dichlorosilane gases were 21 and $20 \mathrm{sccm}$, respectively, in ambient hydrogen. By adding the hydrogen gas at the flow rate of $80 \mathrm{sccm}$, the total gas flow rate was about $100 \mathrm{sccm}$. The total gas flow rate was intentionally made as low as possible in order to achieve a minimum gas consumption. The silicon epitaxial

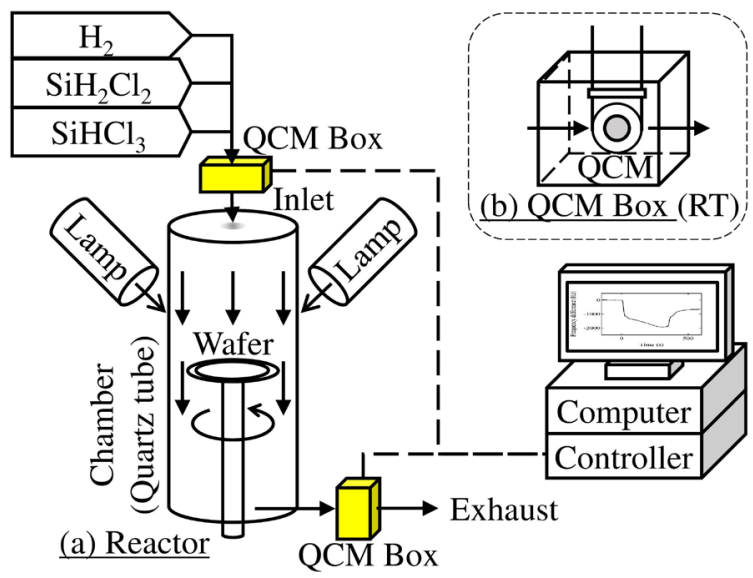

Figure 1. Chemical vapor deposition reactor having two QCM sensors at the inlet and exhaust. (a) Reactor and (b) QCM box.

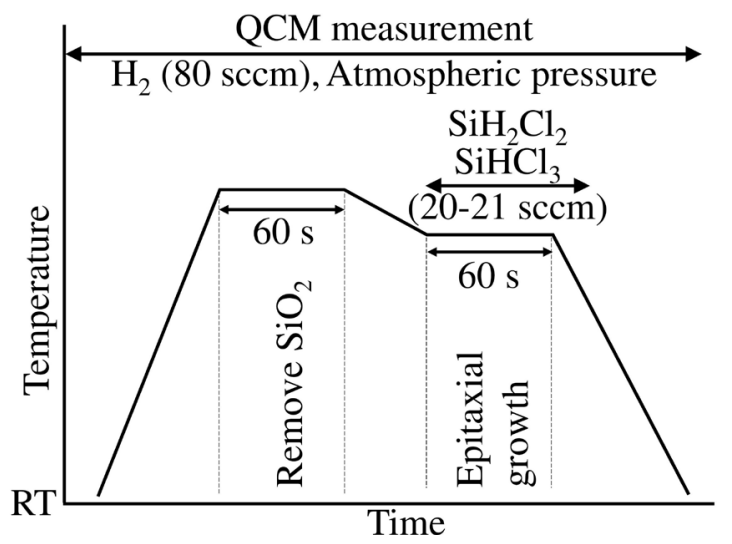

Figure 2. Silicon epitaxial growth process consisting of silicon surface cleaning and silicon epitaxial film deposition. 
film growth rate was evaluated by the increase in the thickness and weight of the wafer.

Various CVD parameters real time influencing the QCM frequency [9] are described by equation (3) and are schematically shown in Figure 3.

$$
\text { QCM frequency change } \propto(\rho \times \mu)^{1.3}
$$

where $\rho$ and $\mu$ are the density and viscosity, respectively, of the gas mixture. The QCM frequency decreases at Step A when the gas density and gas viscosity increase by means of adding a heavy and viscous gas, such as trichlorosilane and dichlorosilane, in ambient hydrogen.

A significantly slow and long decrease at Step B in the QCM frequency, shown in Figure 3, is due to the weight increase, $\Delta \mathrm{w}$, caused by any deposition on the QCM sensor surface [11]. In this study, the byproduct produced by the silicon epitaxial film deposition [12] [19] is assumed to be adsorbed on the QCM sensor surface. At Step C, the QCM frequency increases by means of purging with the hydrogen gas.

\section{Results and Discussion}

\subsection{Overall Behavior}

The typical overall QCM behaviors caused by the dichlorosilane and trichlorosilane gases are shown in Figure 4 and Figure 5, respectively. In these figures, the dark and blue lines are the QCM frequencies measured at the inlet and exhaust, respectively.

The typical QCM frequency behavior is explained by referring to the dark line in Figure 4. Initially, the QCM frequency was confirmed to be in a steady state before introducing the dichlorosilane. After introducing the dichlorosilane gas, the QCM frequency started to decrease at 0 seconds at Step A. This behavior showed the arrival of the dichlorosilane gas at the inlet of the chamber. After the QCM frequency quickly decreased, the QCM frequency gradient became small at Step B. After about 220 seconds, the dichlorosilane gas supply was terminated and was purged by the hydrogen gas. Thus, the QCM frequency began to increase at Step C. In Figure 4 and Figure 5, the QCM frequency values were

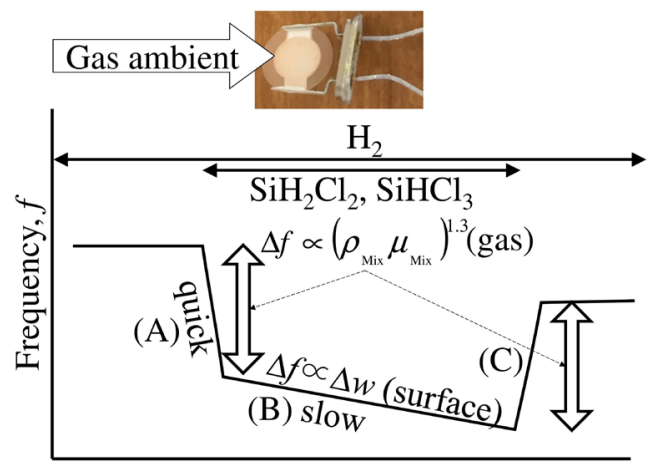

Time, $t$

Figure 3. Parameters changing QCM frequency in this study. 


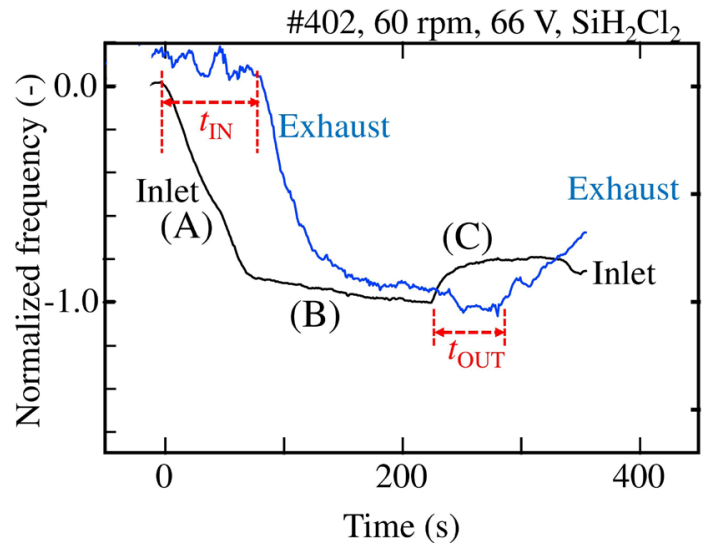

Figure 4. QCM frequency behavior measured at the inlet and exhaust in dichlorosilane-hydrogen system.

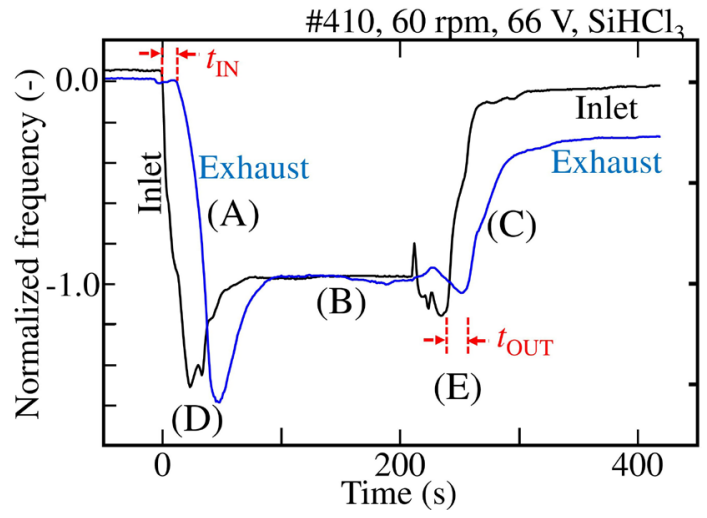

Figure 5. QCM frequency behavior measured at the inlet and exhaust in trichlorosilane-hydrogen system.

normalized using that at Step B.

The QCM frequency at the exhaust showed a behavior similar to that at the inlet, while it was entirely delayed. The QCM frequency at the exhaust started to decrease at $t_{\mathrm{IN}}$ after 0 seconds. The $t_{\mathrm{IN}}$ was the time for the chlorosilanes to arrive at the exhaust after flowing through the chamber. After undergoing the decrease and becoming nearly flat, the QCM frequency started to increase due to the termination of the dichlorosilane gas supply. The time for the QCM frequency at the exhaust to increase was $t_{\mathrm{OUT}}$ seconds delayed from that for the inlet. The typical behavior described above was recognized to be similar to Figure 4 and Figure 5 .

Next, the difference in the behavior between the dichlorosilane and trichlorosilane gases was evaluated. The $t_{\mathrm{IN}}$ and $t_{\mathrm{OUT}}$ values in Figure 4 were nearly 80 and 60 seconds, respectively. These values indicated that nearly one minute was necessary for the dichlorosilane gas to pass through the chamber. In contrast, the $t_{\mathrm{IN}}$ and $t_{\mathrm{OUT}}$ values in Figure 5 were nearly 10 and 20 seconds, respectively. Thus, the trichlorosilane gas could pass through the chamber within 20 seconds. The difference in the QCM frequency between Figure 4 and Figure 5 showed that the trichlorosilane gas motion was faster than that of the dichlorosilane gas. 
In Figure 5, the QCM frequency at the inlet showed a deep-valley-like behavior after 0 and 200 seconds, as denoted by letters D and E, respectively. Similarly, that at the exhaust similarly showed the valley-like changes at the time nearly 20 seconds delayed from those shown at the inlet. Overall in Figure 5, the QCM frequency behavior at the inlet well resembled that at the exhaust. This behavior should be evaluated by studying the gas motions during the introduction and purging processes.

\subsection{Gas Introduction Process}

The behaviors at the introduction of the dichlorosilane and trichlorosilane gases were studied in detail. After evaluating the influence of the wafer rotation, the leading edge motion of the gas was studied.

The periods, $t_{\mathrm{IN}}$, for the dichlorosilane and trichlorosilane gases to flow through the chamber at various wafer rotation rates are shown in Figure 6. The $t_{\mathrm{IN}}$ value of the dichlorosilane gas was widely scattered from 40 to 80 seconds. In contrast, the trichlorosilane gas had lower values between 10 and 20 seconds which scattered in a narrower range than that of the dichlorosilane gas. This trend concluded that the trichlorosilane gas motion through the chamber was faster than that of the dichlorosilane.

Additionally, there seemed to be no dependence of the $t_{\mathrm{IN}}$ value on the wafer rotation rate for both the dichlorosilane and trichlorosilane. The wafer rotation at $4-60 \mathrm{rpm}$ was considered to have no influence on the time for the chlorosilane gases to flow through the chamber.

In order to further evaluate the gas motion, the leading edge motion of the chlorosilanes was studied using the gradient of the QCM frequency immediately after initiating the decrease during Step A. The gradient of the QCM frequency for the dichlorosilane and trichlorosilane gases was obtained using the QCM frequency within 20 and 5 seconds shown in Figure 4 and Figure 5, respectively, after initiating the frequency decrease. Similar to the $t_{\mathrm{IN}}$ values, the QCM frequency gradients both at the inlet and exhaust did not show any relationship with the wafer rotation rate.

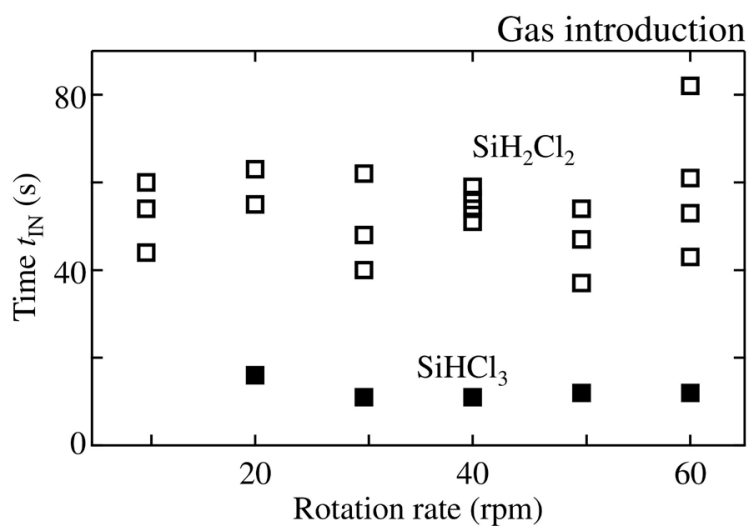

Figure 6. The time, $t_{\mathrm{IN}}$, for dichlorosilane gas and for trichlorosilane gas at various wafer rotation rates. 
Figure 7 shows the relationship of the QCM frequency gradient between the inlet and exhaust. For the trichlorosilane gas denoted by the dark squares in this figure, the QCM frequency gradient at the inlet was distributed in the range between -0.07 and $-0.15 \mathrm{~s}^{-1}$, while those at the exhaust were in the range between -0.03 and $-0.06 \mathrm{~s}^{-1}$. It was noted that the frequency gradient at the exhaust increased with that increasing at the inlet. Thus, the relationship of the QCM frequency between the inlet and exhaust was clearly recognized for the trichlorosilane gas. If the trichlorosilane gas was quickly or moderately introduced at the inlet, such motions directly influenced that at the exhaust. This behavior was recognized in Figure 5.

Next, for the dichlorosilane gas, the QCM frequency gradient was denoted by the open squares in Figure 7. The QCM frequency gradient at the inlet was distributed in the range between -0.1 and $-0.01 \mathrm{~s}^{-1}$, while those at the exhaust were in the range between -0.03 and $-0.01 \mathrm{~s}^{-1}$. This figure shows that the QCM frequency gradient decreased and its scattering range decreased by flowing through the chamber. In addition to this, the QCM frequency gradient at the exhaust was shown to have no relationship to that at the inlet. Thus, the dichlorosilane gas was considered to be well mixed in the chamber; the leading edge motion was different from that of plug flow.

\subsection{Gas Purging Process}

The process for the dichlorosilane and trichlorosilane gases to be purged and replaced with the hydrogen gas was evaluated, because the gas purging process influenced the speed and safety of the process operation. At Step C, the QCM frequency increases, because the gas density and viscosity decreased during purging the chlorosilanes. The QCM frequency gradient was evaluated for 20 and 10 seconds for the dichlorosilane and trichlorosilane gases at Step C.

The dichlorosilane gas behavior was first studied. Figure 8 shows the relationship between the QCM frequency gradient at the inlet and exhaust. The gradient values at the inlet were widely scattered in the range between 0.005 and $0.04 \mathrm{~s}^{-1}$. In contrast, those at the exhaust were $0.002-0.01 \mathrm{~s}^{-1}$. This trend indicated

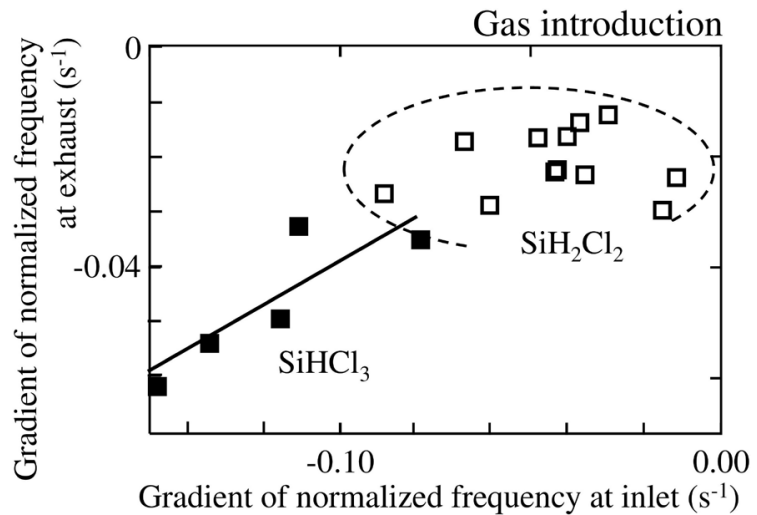

Figure 7. Relationship between gradient of QCM frequency decrease during dichlorosilane and trichlorosilane gas introduction. 
that the purging motion of the dichlorosilane gas appearing at the exhaust was slower than that at the inlet. The fluctuation of the gas flow condition that existed at the inlet could be slightly suppressed by flowing through the chamber. However, this indicated that the purging process for the dichlorosilane gas needed a longer period at the exhaust than that at the inlet. Additionally, the relationship of the QCM frequency behavior between the inlet and the exhaust was not recognized in Figure 8. These conclusions were the same as those obtained from Figure 7.

Similar to Figure 8, the QCM frequency gradient of the trichlorosilane gas was evaluated in Figure 9. The gradient values at the inlet were in the range from 0.03 to $0.35 \mathrm{~s}^{-1}$. Those at the exhaust were in the range from 0.01 to 0.04 $\mathrm{s}^{-1}$. This trend indicated that the purging motion of the trichlorosilane gas appearing at the exhaust was slower than that at the inlet. This conclusion was the same as that for the dichlorosilane. However, in contrast to the dichlorosilane gas, the relationship of the QCM frequency gradient for the trichlorosilane gas between the inlet and exhaust could be clearly recognized in Figure 9 and similar

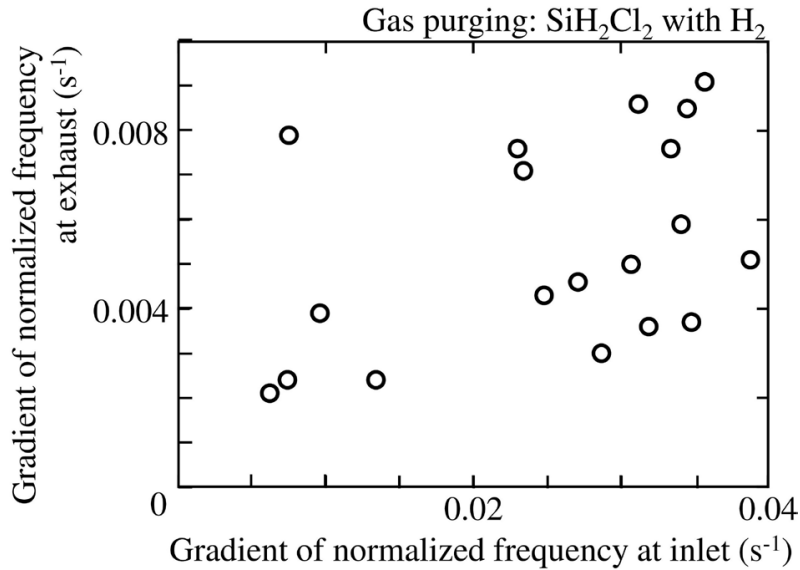

Figure 8. Relationship between gradient of QCM frequency increase during purging dichlorosilane gas with hydrogen gas.

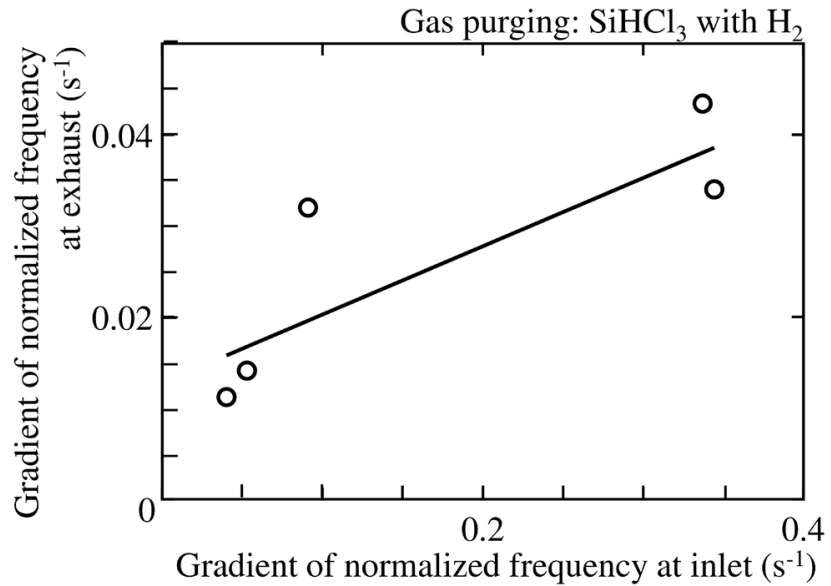

Figure 9. Relationship between gradient of QCM frequency increase during purging trichlorosilane gas with hydrogen gas. 
to that shown in Figure 7.

\subsection{Overall Gas Motion and Process Design}

In this section, the gas motions of the dichlorosilane and trichlorosilane were evaluated from the viewpoint of the impulse and step response methods [20]. As shown in Figure 4, Figures 6-8, the dichlorosilane gas showed a gradual change in the QCM frequency both at the inlet and exhaust. Additionally, any flow fluctuation taking place at the inlet did not influence the gas flow motion at the exhaust. Based on this result, the dichlorosilane gas was considered to be well mixed in the ambient hydrogen in the chamber.

In contrast, the trichlorosilane gas was considered to have a different motion from that of the dichlorosilane gas. The QCM frequency gradient during both the introducing and purging processes of the trichlorosilane gas was clearly greater than that of the dichlorosilane gas. Additionally, the QCM frequency gradient at the exhaust seemed to be influenced by that at the inlet. As shown using letters D and E in Figure 5, the valley-like QCM frequency behavior that appeared at the inlet could be maintained at the exhaust. Taking into account that the valley-like change at the inlet in Figure 5 could be understood as an impulse for the impulse response method [20], any fluctuation at the inlet was considered to be maintained at the exhaust. Thus, the actual flow of the trichlorosilane gas in the chamber was recognized as plug flow [21] [22]. Such a gas motion difference verified the theoretical calculation [13], which showed that the heavy gas flowed down the vertical chamber. Because the slow wafer rotation could locally arrange the flow and heat transport near the wafer, it did not change the entire gas flow.

The Minimal Fab should have a significantly quick production process [7] [12]-[18] because it uses a small-diameter wafer. When the Minimal CVD process uses the trichlorosilane gas, the gas motion from the inlet to the exhaust is expected to be fast during both the introduction and purging processes. The transient process period, such as the gas introduction and gas purging, using the trichlorosilane gas might become several minutes shorter than that using the dichlorosilane gas. Based on this reason, the trichlorosilane gas should be preferred rather than the dichlorosilane gas, if the obtained film qualities were comparable.

\section{Conclusion}

Dichlorosilane gas and trichlorosilane gas in ambient hydrogen were concluded to show different gas flow motions in a slim vertical cold wall CVD reactor for the Minimal Fab based on the evaluation using the two QCM sensors at the inlet and exhaust of the reactor. Typically, the period approaching from the inlet to the exhaust was shorter for the trichlorosilane than that for the dichlorosilane. The trichlorosilane gas was considered to move like a plug flow which might make the transient process operation quick. In contrast, the dichlorosilane gas 
was considered to be well mixed with the gases in the entire chamber. The trichlorosilane gas is shown to achieve a quick epitaxial growth process. The QCM sensor is expected to be a useful in situ real-time tool for improving and controlling the film qualities and the productivities.

\section{Acknowledgements}

The authors thank Mr. Nobuyoshi Enomoto and Mr. Hitoshi Ueno of Halloran Electronics Co., Ltd., for their helpful suggestions and support.

\section{Conflicts of Interest}

The authors declare no conflicts of interest regarding the publication of this paper.

\section{References}

[1] Chang, C.Y. and Sze, S.M. (1996) ULSI Technology. McGraw Hill, New York, USA.

[2] Shimura, F. (1989) Semiconductor Silicon Crystal Technology. Academic Press, San Diego, USA.

[3] Zhang, Y., Zhang, L. and Zhou, C. (2013) Review of Chemical Vapor Deposition of Graphene and Related Applications. Accounts of Chemical Research, 46, 2329-2339. https://doi.org/10.1021/ar300203n

[4] Nakamura, S. (1991) In Situ Monitoring of GaN Growth Using Interference Effects. Japanese Journal of Applied Physics, 30, 1620-1628. https://doi.org/10.1143/JJAP.30.1620

[5] Muroi, M., Yamada, A., Saito, A. and Habuka, H. (2020) Deposition and Etching Behaviour of Boron Trichloride Gas at Silicon Surface. Journal of Crystal Growth, 529, Article ID: 125301. https://doi.org/10.1016/j.jcrysgro.2019.125301

[6] Habuka, H., Aoyama, Y., Akiyama, S., Otsuka, T., Qu, W.F., Shimada, M. and Okuyama, K. (1999) Chemical Process of Silicon Epitaxial Growth in a $\mathrm{SiHCl}_{3}-\mathrm{H}_{2}$ System. Journal of Crystal Growth, 207, 77-86. https://doi.org/10.1016/S0022-0248(99)00360-7

[7] Takahashi, T., Otani, M., Muroi M., Irikura, K., Matsuo, M., Yamada A., Habuka, H., Ishida, Y., Ikeda, S. and Hara, S. (2020) Quartz Crystal Microbalance for Real-Time Monitoring Chlorosilane Gas Transport in Slim Vertical Cold Wall Chemical Vapor Deposition Reactor. Materials Science in Semiconductor Processing, 106, Article ID: 104759. https://doi.org/10.1016/j.mssp.2019.104759

[8] Miyazaki, K., Saito, A. and Habuka, H. (2016) In Situ Measurement for Evaluating Temperature Change Related to Silicon Film Formation in a $\mathrm{SiHCl}_{3}-\mathrm{H}_{2}$ System. ECS Journal of Solid State Science and Technology, 5, 16-20. https://doi.org/10.1149/2.0101602jss

[9] Habuka, H. and Matsui, M. (2013) Langasite Crystal Microbalance Frequency Behavior over Wide Gas Phase Conditions for Chemical Vapor Deposition. Surface and Coatings Technology, 230, 312-315. https://doi.org/10.1016/j.surfcoat.2013.06.052

[10] Habuka, H. and Tanaka, Y. (2013) In-Situ Monitoring of Chemical Vapor Deposition from Trichlorosilane Gas and Monomethylsilane Gas Using Langasite Crystal Microbalance. Journal of Surface Engineered Materials and Advanced Technology, 3, 61-66. https://doi.org/10.4236/jsemat.2013.31A009 
[11] Habuka, H. and Tanaka, Y. (2012) Langasite Crystal Microbalance Used for In-Situ Monitoring of Amorphous Silicon Carbide Film Deposition. ECS Journal of Solid State Science and Technology, 1, 62-65. https://doi.org/10.1149/2.006202jss

[12] Muroi, M., Matsuo, M., Habuka, H., Ishida, Y., Ikeda, S. and Hara, S. (2018) Real Time Evaluation of Silicon Epitaxial Growth Process by Exhaust Gas Measurement Using Quartz Crystal Microbalance. Materials Science in Semiconductor Processing, 88, 192-197. https://doi.org/10.1016/j.mssp.2018.08.014

[13] Irikura, K., Muroi, M., Yamada, A., Matsuo, M., Habuka, H., Ishida, Y., Ikeda, S. and Hara, S. (2018) Advantages of a Slim Vertical Gas Channel at High $\mathrm{SiHCl}_{3}$ Concentrations for Atmospheric Pressure Silicon Epitaxial Growth. Materials Science in Semiconductor Processing, 87, 13-18. https://doi.org/10.1016/j.mssp.2018.07.006

[14] Khumpuang, S., Maekawa, S. and Hara, S. (2013) Photolithography for Minimal Fab System. IEEJ Transactions on Sensors and Micromachines, 133, 272-277. https://doi.org/10.1541/ieejsmas.133.272

[15] Khumpuang, S., Imura, F. and Hara, S. (2015) Analyses on Cleanroom-Free Performance and Transistor Manufacturing Cycle Time of Minimal Fab. IEEE Transactions on Semiconductor Manufacturing, 28, 551-556. https://doi.org/10.1109/TSM.2015.2487324

[16] Li, N., Habuka, H., Ikeda, S. and Hara, S. (2013) Silicon Chemical Vapor Deposition Process Using a Half-Inch Silicon Wafer for Minimal Manufacturing System. Physics Procedia, 46, 230-238. https://doi.org/10.1016/j.phpro.2013.07.059

[17] Li, N., Habuka, H., Ishida, Y., Ikeda, S. and Hara, S. (2016) Reflector Influence on Rapid Heating of Minimal Manufacturing Chemical Vapor Deposition Reactor. ECS Journal of Solid State Science and Technology, 5, 280-284. https://doi.org/10.1149/2.0251605jss

[18] Yamada, A., Li, N., Matsuo, M., Muroi, M., Habuka, H., Ishida, Y., Ikeda, S. and Hara, S. (2017) Transport Phenomena in a Slim Vertical Atmospheric Pressure Chemical Vapor Deposition Reactor Utilizing Natural Convection. Materials Science in Semiconductor Processing, 71, 348-351. https://doi.org/10.1016/j.mssp.2017.08.024

[19] Habuka, H., Sakurai, A. and Saito, A. (2015) By-Product Formation in a Trichlorosilane-Hydrogen System for Silicon Film Deposition. ECS Journal of Solid State Science and Technology, 4, 16-19. http://jss.ecsdl.org/cgi/content/abstract/4/3/P86 https://doi.org/10.1149/2.0031502jss

[20] Kusakabe, K. and Tsukada, T. (2010) Hanno Kogaku. Sankyo, Tokyo. (In Japanese)

[21] Bird, R.B., Stewart, W.E. and Lightfoot, E.N. (2007) Transport Phenomena. 2nd Edition, John Wiley and Sons, Hoboken.

[22] Scott Fogler, H. (2011) Essentials of Chemical Reaction Engineering. Pearson Education, Boston, USA. 